MATHEMATICS OF COMPUTATION

Volume 66, Number 217, January 1997, Pages 373-389

S 0025-5718(97)00807-7

\title{
ON SOME INEQUALITIES FOR THE GAMMA AND PSI FUNCTIONS
}

\author{
HORST ALZER
}

\begin{abstract}
We present new inequalities for the gamma and psi functions, and we provide new classes of completely monotonic, star-shaped, and superadditive functions which are related to $\Gamma$ and $\psi$.
\end{abstract}

Euler's gamma function

$$
\Gamma(x)=\int_{0}^{\infty} e^{-t} t^{x-1} d t \quad(x>0)
$$

is one of the most important functions in analysis and its applications. The history and the development of this function are described in detail in a paper by P. J. Davis [10].

There exists a very extensive literature on the gamma function. In particular, numerous remarkable inequalities involving $\Gamma$ and its logarithmic derivative $\psi=$ $\Gamma^{\prime} / \Gamma$ have been published by different authors; see, e.g., [2], [3], [6], [7], [9], [12], [13], [18]-[27], [29]-[33], [35]-[46], [50]. Many of these inequalities follow immediately from the monotonicity properties of functions which are closely related to $\Gamma$ and $\psi$. In several recent papers [2], [9], [24], [39] it is proved that these functions are not only monotonic, but even completely monotonic. We recall that a function $f$ is said to be completely monotonic on an interval $I$ if $f$ has derivatives of all orders on $I$ which alternate successively in sign, that is,

$$
(-1)^{n} f^{(n)}(x) \geq 0
$$

for all $x \in I$ and for all $n \geq 0$. If inequality (1.1) is strict for all $x \in I$ and all $n \geq 0$, then $f$ is said to be strictly completely monotonic.

It is known that completely monotonic functions play an eminent role in areas like probability theory [15], numerical analysis [49], physics [11], and the theory of special functions. For instance, M. E. Muldoon [39] showed how the notation of complete monotonicity can be used to characterize the gamma function. An interesting exposition of the main results on completely monotonic functions is given in [48].

"In view of the importance of completely monotonic functions ... it may be of interest to add to the available list of such functions" $[24$, p. 1]. It is the main

Received by the editor October 13, 1995 and, in revised form, March 4, 1996.

1991 Mathematics Subject Classification. Primary 33B15; Secondary 26D07.

Key words and phrases. Gamma function, psi function, complete monotonicity, inequalities, star-shaped functions, super-additive functions, infinite divisibility, Laplace transform.

(C)1997 American Mathematical Society 
purpose of this paper to present new classes of completely monotonic functions which are all closely related to the gamma and psi functions. Applications of our monotonicity theorems lead to new inequalities for $\Gamma$ and $\psi$. Furthermore, we extend and sharpen known inequalities due to W. Gautschi, H. Minc and L. Sathre, and others, and we provide new classes of star-shaped and super-additive functions. In the final section we apply one of our results to present functions which are Laplace transforms of infinitely divisible probability measures.

\section{2}

In a recently published article G. D. Anderson et al. [3] proved that the function $f(x)=x(\log (x)-\psi(x))$ is strictly decreasing and strictly convex on $(0, \infty)$. Moreover, the authors presented (complicated) proofs for

$$
\lim _{x \rightarrow 0} f(x)=1 \text { and } \quad \lim _{x \rightarrow \infty} f(x)=1 / 2 .
$$

We note that the limits (2.1) follow immediately from the representations

$$
f(x)=x \log (x)-x \psi(x+1)+1
$$

and

$$
f(x)=\frac{1}{2}+\frac{1}{12 x}-\frac{\theta}{120 x^{3}} \quad(0<\theta<1)
$$

see $[16$, p. 824].

From (2.1) and the monotonicity of $f$ we conclude

$$
\frac{1}{2 x}<\log (x)-\psi(x)<\frac{1}{x} \quad(x>0) .
$$

This extends a result of H. Minc and L. Sathre [37], who established (2.2) for $x>1$, and used it to prove several discrete inequalities involving the geometric mean of the first $n$ positive integers. Refinements of (2.2) were given by L. Gordon [22]. Our first theorem provides an extension of the result given by Anderson et al.; we prove that $f$ is not only decreasing and convex, but even completely monotonic.

Theorem 1. Let $\alpha$ be a real number. The function

$$
f_{\alpha}(x)=x^{\alpha}(\log (x)-\psi(x))
$$

is strictly completely monotonic on $(0, \infty)$ if and only if $\alpha \leq 1$.

Proof. First, we show that $f_{1}$ is strictly completely monotonic on $(0, \infty)$. Using Binet's formula [14, p. 18] we obtain the representation

$$
f_{1}(x)=x \int_{0}^{\infty} \varphi(t) e^{-t x} d t
$$

where

$$
\varphi(t)=1 /\left(1-e^{-t}\right)-1 / t
$$

Easy computations reveal that the function $\varphi$ is strictly increasing on $(0, \infty)$ with $\lim _{t \rightarrow 0} \varphi(t)=1 / 2$ and $\lim _{t \rightarrow \infty} \varphi(t)=1$. 
Let $n \geq 1$; from (2.3) we get

$$
\begin{aligned}
(-1)^{n} f_{1}^{(n)}(x)= & x(-1)^{n} \frac{d^{n}}{d x^{n}} \int_{0}^{\infty} \varphi(t) e^{-t x} d t \\
& \quad-n(-1)^{n-1} \frac{d^{n-1}}{d x^{n-1}} \int_{0}^{\infty} \varphi(t) e^{-t x} d t \\
= & x \int_{0}^{\infty} \varphi(t) e^{-t x} t^{n} d t-n \int_{0}^{\infty} \varphi(t) e^{-t x} t^{n-1} d t \\
= & \int_{0}^{n / x} \varphi(t) e^{-t x} t^{n-1}(t x-n) d t+\int_{n / x}^{\infty} \varphi(t) e^{-t x} t^{n-1}(t x-n) d t .
\end{aligned}
$$

If $0<t<n / x$, then we obtain $\varphi(t)<\varphi(n / x)$; and if $n / x<t$, then we have $\varphi(n / x)<\varphi(t)$. Hence, from (2.4) we get

$$
\begin{aligned}
(-1)^{n} f_{1}^{(n)}(x)>\varphi(n / x) & \int_{0}^{n / x} e^{-t x} t^{n-1}(t x-n) d t \\
& +\varphi(n / x) \int_{n / x}^{\infty} e^{-t x} t^{n-1}(t x-n) d t \\
= & \varphi(n / x) \int_{0}^{\infty} e^{-t x} t^{n-1}(t x-n) d t .
\end{aligned}
$$

Using

$$
\int_{0}^{\infty} e^{-t x} t^{m} d t=(m !) / x^{m+1} \quad(x>0 ; m=0,1,2, \ldots),
$$

we conclude

$$
\int_{0}^{\infty} e^{-t x} t^{n-1}(t x-n) d t=0
$$

so that (2.5) implies

$$
(-1)^{n} f_{1}^{(n)}(x)>0 \text { for } x>0 \text { and } n=0,1,2, \ldots .
$$

From Leibniz' rule

$$
(-1)^{n}(u(x) v(x))^{(n)}=\sum_{i=0}^{n}\left(\begin{array}{c}
n \\
i
\end{array}\right)(-1)^{i} u^{(i)}(x)(-1)^{n-i} v^{(n-i)}(x),
$$

it follows that the product of two strictly completely monotonic functions is also strictly completely monotonic. Since $u_{\alpha}(x)=x^{\alpha-1}(\alpha<1)$ is strictly completely monotonic on $(0, \infty)$, we conclude that $f_{\alpha}(x)=u_{\alpha}(x) f_{1}(x)(\alpha \leq 1)$ has the same property.

Next, we assume that $f_{\alpha}$ is strictly completely monotonic on $(0, \infty)$. Then we have for all $x>0$ :

$$
f_{\alpha}^{\prime}(x)=x^{\alpha-1}\left[\alpha(\log (x)-\psi(x))+1-x \psi^{\prime}(x)\right]<0,
$$

which implies

$$
\alpha<\frac{x^{2} \psi^{\prime}(x)-x}{x(\log (x)-\psi(x))} .
$$

If we let $x$ tend to 0 , then we get $\alpha \leq 1$. The proof of Theorem 1 is complete. 
Anderson et al. [3] used the monotonicity of $f_{1}$ to prove that the function $g_{1}(x)=$ $x^{1 / 2}(e / x)^{x} \Gamma(x)$ is decreasing on $(0, \infty)$, and that $g_{2}(x)=x(e / x)^{x} \Gamma(x)$ is increasing on $(0, \infty)$. The following theorem provides a slight extension of these results.

Theorem 2. Let $a \geq 0, r$ and $s$ be real numbers. The function

$$
F_{r}(x)=x^{r}(e / x)^{x} \Gamma(x)
$$

is decreasing on $(a, \infty)$ if and only if $r \leq 1 / 2$; and the function

$$
G_{s}(x)=x^{s}(e / x)^{x} \Gamma(x)
$$

is increasing on $(a, \infty)$ if and only if

$$
s \geq \begin{cases}a(\log (a)-\psi(a)) & \text { if } a>0, \\ 1 & \text { if } a=0 .\end{cases}
$$

Proof. Since $F_{r}^{\prime}(x) \leq 0$ is equivalent to

$$
r \leq x(\log (x)-\psi(x))=f_{1}(x),
$$

the first part of Theorem 2 follows from the fact that $f_{1}$ is decreasing on $(0, \infty)$ and tends to $1 / 2$ if $x$ tends to $\infty$. The second part can be proved similarly. We omit the details.

Remark. Let $g$ be a strictly completely monotonic function on $(0, \infty)$, and let $c$ be a real number. From Theorem 1 we conclude that the function

$$
x \mapsto g(x)\left(f_{1}(x)-c\right)
$$

is strictly completely monotonic on $(0, \infty)$ if and only if $c \leq 1 / 2$. This extends a result of M. E. Muldoon [39], who proved the complete monotonicity of (2.6) for the special case $g(x)=1 / x$.

In 1974, C. H. Kimberling [28] established the following property of completely monotonic functions: If $f$ is continuous on $[0, \infty)$ and completely monotonic on $(0, \infty)$ and satisfies $0<f(x) \leq 1$ for all $x \geq 0$, then $\log (f)$ is super-additive on $[0, \infty)$.

We recall that a function $g$ is said to be super-additive on an interval $I$ if

$$
g(x)+g(y) \leq g(x+y) \quad \text { for all } x, y \in I \text { with } x+y \in I .
$$

In the previous section we have proved that $f(x)=x(\log (x)-\psi(x))$ is continuous on $[0, \infty)$, completely monotonic on $(0, \infty)$, and $1 / 2<f(x) \leq 1$ for all $x \geq 0$, so that Kimberling's theorem implies

$$
1 \leq \frac{f(x+y)}{f(x) f(y)} \quad(x, y \geq 0) .
$$

This leads to the problem to determine sharp upper and lower bounds for the ratio $f(x+y) /(f(x) f(y))$.

Theorem 3. Let $f(x)=x(\log (x)-\psi(x))$. Then we have for all real $x, y \geq 0$ :

$$
1 \leq \frac{f(x+y)}{f(x) f(y)}<2
$$

Both bounds are best possible. 
Proof. To prove the second inequality of (3.1) we define

$$
g(x, y)=f(x+y) / f(x) .
$$

Partial differentiation yields

$$
\frac{\partial g(x, y)}{\partial x}=\frac{f(x+y)}{f(x)}\left[\frac{f^{\prime}(x+y)}{f(x+y)}-\frac{f^{\prime}(x)}{f(x)}\right] .
$$

Let

$$
h(x, y)=f^{\prime}(x+y) / f(x+y)
$$

then we have

$$
\frac{\partial h(x, y)}{\partial y}=\left[f^{\prime \prime}(x+y) f(x+y)-\left(f^{\prime}(x+y)\right)^{2}\right] /(f(x+y))^{2} .
$$

Since completely monotonic functions are log-convex (see [17]), we conclude from (3.3) and Theorem 1 that $\partial h(x, y) / \partial y \geq 0$. This implies

$$
h(x, y) \geq h(x, 0),
$$

so that (3.2) and (3.4) lead to

$$
\frac{\partial g(x, y)}{\partial x} \geq 0 \quad \text { and } \quad g(x, y) \leq \lim _{x \rightarrow \infty} g(x, y)=1 .
$$

Thus, we have

$$
\frac{f(x+y)}{f(x)} \leq 1<2 f(y) \quad \text { for } x, y \geq 0
$$

From

$$
\lim _{y \rightarrow 0} \frac{f(x+y)}{f(x) f(y)}=1
$$

and

$$
\lim _{y \rightarrow \infty} \lim _{x \rightarrow \infty} \frac{f(x+y)}{f(x) f(y)}=\lim _{y \rightarrow \infty} \frac{1}{f(y)}=2,
$$

we conclude that both bounds in (3.1) are sharp.

Remark. If we set

$$
Q_{\alpha}(x, y)=f_{\alpha}(x+y) /\left(f_{\alpha}(x) f_{\alpha}(y)\right)
$$

where $f_{\alpha}(x)=x^{\alpha}(\log (x)-\psi(x))$ and $\alpha \neq 1$, then we conclude from the limit relations

$$
\lim _{y \rightarrow 0} \lim _{x \rightarrow \infty} Q_{\alpha}(x, y)=\lim _{y \rightarrow 0} \frac{1}{f_{\alpha}(y)}= \begin{cases}\infty & \text { if } \alpha>1 \\ 0 & \text { if } \alpha<1\end{cases}
$$

and

$$
\lim _{y \rightarrow \infty} \lim _{x \rightarrow \infty} Q_{\alpha}(x, y)=\lim _{y \rightarrow \infty} \frac{1}{f_{\alpha}(y)}= \begin{cases}0 & \text { if } \alpha>1, \\ \infty & \text { if } \alpha<1,\end{cases}
$$

that the inequalities $0 \leq Q_{\alpha}(x, y)<\infty(x, y>0 ; \alpha \neq 1)$ cannot be refined. 
In 1974, W. Gautschi [20] proved that the function $x \mapsto x \psi(x)$ is convex on $(0, \infty)$, and applied this result to establish some mean value inequalities involving the gamma function. Our next theorem provides an extension of Gautschi's proposition.

Theorem 4. Let $n \geq 2$ be an integer. Then we have for all real $x>0$ :

$$
0<(-1)^{n} x^{n-1}[x \psi(x)]^{(n)}<(n-2) ! .
$$

Both bounds are best possible.

Proof. Let $f(x)=x(\log (x)-\psi(x))$ and let $n \geq 2$. From Theorem 1 we obtain

$$
\begin{aligned}
0 & <(-1)^{n} f^{(n)}(x)=(-1)^{n}(x \log (x))^{(n)}-(-1)^{n}(x \psi(x))^{(n)} \\
& =\frac{(n-2) !}{x^{n-1}}-(-1)^{n}(x \psi(x))^{(n)},
\end{aligned}
$$

which leads to the second inequality of (4.1). Since

$$
\psi^{(m)}(x)=(-1)^{m+1} m ! \sum_{i=0}^{\infty} \frac{1}{(x+i)^{m+1}} \quad(m=1,2, \ldots),
$$

we get

$$
\begin{aligned}
(-1)^{n}(x \psi(x))^{(n)} & =(-1)^{n}\left[x \psi^{(n)}(x)+n \psi^{(n-1)}(x)\right] \\
& =n ! \sum_{i=1}^{\infty} \frac{i}{(x+i)^{n+1}}>0,
\end{aligned}
$$

which implies the left-hand inequality of (4.1).

It remains to show that the bounds in (4.1) cannot be refined. Using $\psi^{(m)}(x)=$ $\psi^{(m)}(x+1)+(-1)^{m+1} m ! / x^{m+1}(m=0,1, \ldots)$, we get

$$
(-1)^{n} x^{n-1}(x \psi(x))^{(n)}=(-1)^{n} x^{n-1}\left[x \psi^{(n)}(x+1)+n \psi^{(n-1)}(x+1)\right] .
$$

Hence, we have

$$
\lim _{x \rightarrow 0}(-1)^{n} x^{n-1}(x \psi(x))^{(n)}=0 .
$$

Let $m \geq 1$ be an integer; from

$$
\begin{aligned}
\frac{1}{m x^{m}} & =\int_{0}^{\infty} \frac{d t}{(x+t)^{m+1}} \leq \sum_{i=0}^{\infty} \frac{1}{(x+i)^{m+1}} \leq \frac{1}{x^{m+1}}+\int_{0}^{\infty} \frac{d t}{(x+t)^{m+1}} \\
& =\frac{1}{x^{m+1}}+\frac{1}{m x^{m}},
\end{aligned}
$$

we conclude

$$
\begin{aligned}
(m-1) ! & \leq m ! x^{m} \sum_{i=0}^{\infty} \frac{1}{(x+i)^{m+1}}=-(-1)^{m} x^{m} \psi^{(m)}(x) \\
& \leq \frac{m !}{x}+(m-1) !
\end{aligned}
$$

which implies

$$
\lim _{x \rightarrow \infty}(-1)^{m} x^{m} \psi^{(m)}(x)=-(m-1) ! \quad(m \geq 1) .
$$


From (4.2) and (4.3) we obtain

$$
\lim _{x \rightarrow \infty}(-1)^{n} x^{n-1}(x \psi(x))^{(n)}=(n-2) ! .
$$

Hence, both bounds in (4.1) are best possible.

A function $f$ is said to be star-shaped on $(0, \infty)$ if

$$
f(a x) \leq a f(x)
$$

is valid for all $x>0$ and for all $a \in(0,1)$. These functions have been investigated intensively by A. M. Bruckner and E. Ostrow [8]. It is well known that starshaped functions are super-additive. Indeed, from (5.1) we obtain $f(x) \leq$ $(x /(x+y)) f(x+y)$ and $f(y) \leq(y /(x+y)) f(x+y)$; summing leads to $f(x)+$ $f(y) \leq f(x+y)$. In this section we answer the questions: For which real $\beta$ is

$$
x \mapsto \frac{(-1)^{k+1} x^{\beta}}{\psi^{(k)}(x)-(\log (x))^{(k)}} \quad(0 \leq k \in \mathbb{Z})
$$

star-shaped; and for which $\beta$ is this function super-additive?

Theorem 5. Let $k \geq 0$ be an integer and let $\beta$ be a real number. The function

$$
x \mapsto g_{\beta}(k ; x)=\frac{(-1)^{k+1} x^{\beta}}{\psi^{(k)}(x)-(\log (x))^{(k)}}
$$

is star-shaped on $(0, \infty)$ if and only if $\beta \geq-k$.

Proof. Let $g_{\beta}$ be star-shaped on $(0, \infty)$. We assume (for a contradiction) that $\beta<-k$. We consider two cases. If $k=0$, then inequality

$$
g_{\beta}(0 ; a x) \leq a g_{\beta}(0 ; x) \quad(x>0 ; 0<a<1)
$$

and Theorem 1 imply that

$$
0<\log (x)-\psi(x) \leq \frac{a^{-\beta}}{x}(a x)[\log (a x)-\psi(a x)] .
$$

If we let $a$ tend to 0 , then we conclude from $\beta<0$ that the product on the righthand side of (5.2) tends to 0 . Let $k \geq 1$; from

$$
\begin{aligned}
(-1)^{k+1} \psi^{(k)}(x) & =k ! \sum_{i=0}^{\infty} \frac{1}{(x+i)^{k+1}}>k ! \int_{0}^{\infty} \frac{d t}{(x+t)^{k+1}} \\
& =\frac{(k-1) !}{x^{k}}=(-1)^{k+1}(\log (x))^{(k)}
\end{aligned}
$$

and

$$
g_{\beta}(k ; a x) \leq a g_{\beta}(k ; x)
$$

we obtain

$$
\begin{aligned}
0 & <x^{-\beta}\left[(-1)^{k+1} \psi^{(k)}(x)-\frac{(k-1) !}{x^{k}}\right] \\
\leq & \frac{1}{x}\left[(-1)^{k+1}(a x)^{1-\beta} \psi^{(k)}(a x)-(k-1) !(a x)^{1-\beta-k}\right] \\
=\frac{1}{x}\left[(-1)^{k+1}(a x)^{1-\beta} \psi^{(k)}(a x+1)\right. & +k !(a x)^{-\beta-k} \\
& \left.-(k-1) !(a x)^{1-\beta-k}\right] .
\end{aligned}
$$


Since $\beta<-k$, we conclude that each term on the right-hand side of (5.4) tends to 0 if $a$ tends to 0 . Hence, if $g_{\beta}$ is star-shaped on $(0, \infty)$, then $\beta \geq-k$.

Next, we assume that $\beta \geq-k$; to prove

$$
g_{\beta}(k ; a x) \leq a g_{\beta}(k ; x)
$$

for $x>0$ and $a \in(0,1)$, we reconsider two cases.

Case 1: $k=0$. Then inequality (5.5) is equivalent to

$$
\log (x)-\psi(x) \leq a^{1-\beta}[\log (a x)-\psi(a x)]=F(a), \quad \text { say } .
$$

It suffices to show that $F$ is decreasing on $(0,1]$. We obtain

$$
a^{\beta} F^{\prime}(a)=(1-\beta)[\log (a x)-\psi(a x)]+1-(a x) \psi^{\prime}(a x) .
$$

If we set

$$
G(z)=(1-\beta)[\log (z)-\psi(z)]+1-z \psi^{\prime}(z) \quad(z>0),
$$

then we conclude from (5.3) (with $k=1$ ) and the right-hand side inequality of (4.1) (with $n=2$ ) that

$$
G^{\prime}(z)=\beta\left(\psi^{\prime}(z)-1 / z\right)+1 / z-(z \psi(z))^{\prime \prime}>0 .
$$

From (2.2) and (4.3) we get

$$
G(z)<\lim _{z \rightarrow \infty} G(z)=0,
$$

which implies $F^{\prime}(a)<0$ for all $a \in(0,1]$.

Case $2: k \geq 1$. Then inequality (5.5) can be written as

$$
H(1) \leq H(a),
$$

where

$$
H(a)=a^{1-\beta}\left[(-1)^{k+1} \psi^{(k)}(a x)-(k-1) ! /(a x)^{k}\right] .
$$

Differentiation yields

$$
\begin{aligned}
a^{\beta} H^{\prime}(a)= & (1-\beta)\left[(-1)^{k+1} \psi^{(k)}(a x)-(k-1) ! /(a x)^{k}\right] \\
& +(-1)^{k+1} a x \psi^{(k+1)}(a x)+k ! /(a x)^{k} .
\end{aligned}
$$

We replace $a x$ by $z$ and denote the right-hand side of (5.7) by $J(z)$. Then we obtain

$$
\begin{aligned}
J^{\prime}(z)= & (1-\beta)\left[(-1)^{k+1} \psi^{(k+1)}(z)+k ! / z^{k+1}\right] \\
& +(-1)^{k+1} \psi^{(k+1)}(z)+(-1)^{k+1} z \psi^{(k+2)}(z)-k ! k / z^{k+1} .
\end{aligned}
$$

From the second inequality of (4.1) we obtain

$$
\begin{aligned}
k ! / z^{k+1} & >(-1)^{k}(z \psi(z))^{(k+2)} \\
& =(-1)^{k}\left[z \psi^{(k+2)}(z)+(k+2) \psi^{(k+1)}(z)\right] .
\end{aligned}
$$

Using (5.3), (5.8), and (5.9) we get

$$
J^{\prime}(z)>(\beta+k)\left[(-1)^{k} \psi^{(k+1)}(z)-k ! / z^{k+1}\right] \geq 0 .
$$

Thus, $J$ is strictly increasing on $(0, \infty)$. From (4.3) we conclude that $\lim _{z \rightarrow \infty} z^{k} J(z)$ $=0$, which implies that $J(z) \leq 0$ for all $z>0$. Therefore, $H$ in decreasing on $(0,1]$ which leads to inequality (5.6). This completes the proof of Theorem 5. 
Theorem 6. Let $k \geq 0$ be an integer and let $\beta$ be a real number. The function

$$
x \mapsto g_{\beta}(k ; x)=\frac{(-1)^{k+1} x^{\beta}}{\psi^{(k)}(x)-(\log (x))^{(k)}}
$$

is super-additive on $(0, \infty)$ if and only if $\beta \geq-k$.

Proof. If $\beta \geq-k$, then we conclude from Theorem 5 that $g_{\beta}$ is star-shaped, which implies that $g_{\beta}$ is super-additive. Next, we suppose that

$$
g_{\beta}(k ; x)+g_{\beta}(k ; y) \leq g_{\beta}(k ; x+y)
$$

holds for all $x, y>0$. We set in (5.10) $x=y$ and obtain after simple manipulations

$$
2^{-\beta} \leq \frac{x(\log (x)-\psi(x))}{2 x(\log (2 x)-\psi(2 x))} \quad \text { if } k=0,
$$

and

$$
2^{-\beta-k} \leq \frac{(-1)^{k} x^{k+1} \psi^{(k)}(x+1)+(k-1) ! x-k !}{(-1)^{k}(2 x)^{k+1} \psi^{(k)}(2 x+1)+(k-1) !(2 x)-k !} \quad \text { if } k \geq 1 .
$$

If we let $x$ tend to 0 , then we obtain $\beta \geq-k$.

Remark. In 1989, S. Y. Trimble et al. [47] introduced an interesting subclass of the completely monotonic functions. A function $g$ is called strongly completely monotonic on $(0, \infty)$ if

$$
x \mapsto(-1)^{n} x^{n+1} g^{(n)}(x)
$$

is nonnegative and decreasing on $(0, \infty)$ for $n=0,1,2, \ldots$. The authors showed that these functions have a close connection to star-shaped functions. Indeed, one of their results states: If $g$ is strongly completely monotonic on $(0, \infty)$ and $g \not \equiv 0$, then $1 / g$ is star-shaped.

In the past many articles were published providing different inequalities for the ratio $\Gamma(x+1) / \Gamma(x+s)$, where $x>0$ and $s \in(0,1)$; see, e.g., [2], [13], [18], [25], [26], [29]-[31], [45], [50]. In this section we present upper and lower bounds for the difference $\psi(x+1)-\psi(x+s)$. In 1972, Y. L. Luke [33] considered the special case $s=1 / 2$. He pointed out that this difference can be represented in terms of Gauss' hypergeometric series

$$
{ }_{2} F_{1}(a, b ; c ; z)=\sum_{n=0}^{\infty} \frac{(a)_{n}(b)_{n}}{(c)_{n}} \frac{z^{n}}{n !},
$$

where $(a)_{n}=\Gamma(a+n) / \Gamma(a)$, namely,

$$
\psi(x+1)-\psi(x+1 / 2)=\frac{1}{x+1 / 2}{ }_{2} F_{1}(1,2 x+1 ; 2 x+2 ;-1),
$$

and used well-known Padé-approximation for ${ }_{2} F_{1}$ to obtain rational bounds for $\psi(x+1)-\psi(x+1 / 2)$. By using a different approach we get the following sharp inequalities for $\psi(x+1)-\psi(x+s)$. 
Theorem 7. Let $n \geq 0$ be an integer and let $x>0$ and $s \in(0,1)$ be real numbers. Then we have

$$
A_{n}(s ; x)<\psi(x+1)-\psi(x+s)<A_{n}(s ; x)+\delta_{n}(s ; x),
$$

where

$$
A_{n}(s ; x)=(1-s)\left[\frac{1}{x+s+n}+\sum_{i=0}^{n-1} \frac{1}{(x+i+1)(x+i+s)}\right]
$$

and

$$
\delta_{n}(s ; x)=\frac{1}{x+n+s} \log \frac{(x+n)^{(x+n)(1-s)}(x+n+1)^{(x+n+1) s}}{(x+n+s)^{x+n+s}} .
$$

Proof. From Theorem 4 we conclude that the function $h(x)=x \psi(x)$ is strictly convex on $(0, \infty)$. If we set in Jensen's inequality

$$
h(s u+(1-s) v)<\operatorname{sh}(u)+(1-s) h(v) \quad(u, v>0 ; u \neq v ; 0<s<1),
$$

$u=x+1$ and $v=x$, and make use of the identity $\psi(x+1)-\psi(x)=1 / x$, then we get

$$
\frac{1-s}{x+s}<\psi(x+1)-\psi(x+s) .
$$

Next, we replace in (6.2) $x$ by $x+1$ and obtain the following sharpening of (6.2):

$$
\frac{1-s}{x+s+1}+\frac{1-s}{(x+1)(x+s)}<\psi(x+1)-\psi(x+s) .
$$

Repeating this process $n$ times we get

$$
\frac{1-s}{x+s+n}+(1-s) \sum_{i=0}^{n-1} \frac{1}{(x+i+1)(x+i+s)}<\psi(x+1)-\psi(x+s),
$$

that is, the left-hand inequality of (6.1). Using the same method of proof with $\tilde{h}(x)=x(\log (x)-\psi(x))$ instead of $h$, we obtain the second inequality of (6.1). We omit the details.

Remark. A simple calculation shows that $\lim _{n \rightarrow \infty} \delta_{n}(s ; x)=0$.

\section{7}

In 1964, H. Minc and L. Sathre [37] proved that the inequalities

$$
0<\log \Gamma(x)-\left(x-\frac{1}{2}\right) \log (x)+x-\frac{1}{2} \log (2 \pi)<\frac{1}{x}
$$

are valid for $x>1$. Since the function $\log \Gamma(x)$ is asymptotically equal to the (divergent) series

$$
\left(x-\frac{1}{2}\right) \log (x)-x+\frac{1}{2} \log (2 \pi)+\sum_{i=1}^{\infty} \frac{B_{2 i}}{2 i(2 i-1) x^{2 i-1}},
$$

where $B_{i}(i=0,1,2, \ldots)$ are Bernoulli numbers, defined by

$$
\frac{t}{e^{t}-1}=\sum_{i=0}^{\infty} B_{i} \frac{t^{i}}{i !}
$$


(see $[16$, p. 823$]$ ), it is natural to ask whether it is possible to determine the sign of

$$
\begin{aligned}
S_{k}(x)= & \log \Gamma(x)-\left(x-\frac{1}{2}\right) \log (x)+x-\frac{1}{2} \log (2 \pi) \\
& -\sum_{i=1}^{k} \frac{B_{2 i}}{2 i(2 i-1) x^{2 i-1}} \quad(0 \leq k \in \mathbb{Z}) .
\end{aligned}
$$

As by-products of the next theorem we obtain sgn $S_{k}(x)=(-1)^{k}$ for $x>0$ and $k \geq 0$, and we get that (7.1) (with the upper bound $1 /(12 x)$ ) holds for all $x>0$. Further refinements of (7.1) can be found in [22].

Muldoon [39] investigated $S_{0}(x)$ and proved that this function is completely monotonic on $(0, \infty)$. This result can be extended:

Theorem 8. Let $n \geq 0$ be an integer. The functions

$$
F_{n}(x)=\log \Gamma(x)-\left(x-\frac{1}{2}\right) \log (x)+x-\frac{1}{2} \log (2 \pi)-\sum_{i=1}^{2 n} \frac{B_{2 i}}{2 i(2 i-1) x^{2 i-1}}
$$

and

$$
G_{n}(x)=-\log \Gamma(x)+\left(x-\frac{1}{2}\right) \log (x)-x+\frac{1}{2} \log (2 \pi)+\sum_{i=1}^{2 n+1} \frac{B_{2 i}}{2 i(2 i-1) x^{2 i-1}}
$$

are strictly completely monotonic on $(0, \infty)$.

Proof. We only establish that $F_{n}$ is strictly completely monotonic; the proof for $G_{n}$ is similar. In [16, pp. 823-824] the following representations for $F_{n}$ and $F_{n}^{\prime}$ are given:

$$
F_{n}(x)=\frac{B_{4 n+2}}{(4 n+1)(4 n+2)} \frac{\theta}{x^{4 n+1}} \quad(0<\theta<1)
$$

and

$$
F_{n}^{\prime}(x)=-\frac{B_{4 n+2}}{4 n+2} \frac{\tilde{\theta}}{x^{4 n+2}} \quad(0<\tilde{\theta}<1)
$$

Since $B_{4 n+2}>0$ (see [4, p. 267]), we obtain $F_{n}(x)>0$ and $F_{n}^{\prime}(x)<0$ for $x>0$. Let $k \geq 1$; differentiation yields

$$
\begin{aligned}
\frac{1}{k !}(-1)^{k+1} F_{n}^{(k+1)}(x)= & \sum_{i=0}^{\infty} \frac{1}{(x+i)^{k+1}}-\frac{1}{k x^{k}}-\frac{1}{2 x^{k+1}} \\
& +\frac{(-1)^{k+1}}{k !} \sum_{i=1}^{2 n}\left[\frac{B_{2 i}}{2 i} \prod_{j=0}^{k-1}(-2 i-j)\right] \frac{1}{x^{2 i+k}} .
\end{aligned}
$$


To find a lower bound for this sum we make use of Euler's summation formula [1, p. 806]:

$$
\begin{aligned}
\sum_{i=0}^{p} f(a+i)= & \int_{a}^{b} f(t) d t+\frac{1}{2}(f(a)+f(b)) \\
& +\sum_{i=1}^{m} \frac{B_{2 i}}{(2 i) !}\left(f^{(2 i-1)}(b)-f^{(2 i-1)}(a)\right) \\
& +\frac{B_{2 m+2}}{(2 m+2) !} \sum_{i=0}^{p-1} f^{(2 m+2)}(a+i+\theta)
\end{aligned}
$$

where $b=a+p$ and $\theta \in(0,1)$. We set $f(x)=1 / x^{k+1}, a=x$, and $m=2 n$ in (7.3) and let $p$ tend to $\infty$. Then we obtain

$$
\begin{aligned}
\sum_{i=0}^{\infty} \frac{1}{(x+i)^{k+1}}= & \frac{1}{k x^{k}}+\frac{1}{2 x^{k+1}}-\sum_{i=1}^{2 n}\left[\frac{B_{2 i}}{(2 i) !} \prod_{j=0}^{2 i-2}(-k-1-j)\right] \frac{1}{x^{2 i+k}} \\
& +\frac{B_{4 n+2}}{(4 n+2) !}\left[\prod_{j=0}^{4 n+1}(-k-1-j)\right] \sum_{i=0}^{\infty} \frac{1}{(x+\theta+i)^{4 n+k+3}} .
\end{aligned}
$$

Using $B_{4 n+2}>0$ and $\prod_{j=0}^{4 n+1}(-k-1-j)>0$ we get from (7.4):

$$
\sum_{i=0}^{\infty} \frac{1}{(x+i)^{k+1}}>\frac{1}{k x^{k}}+\frac{1}{2 x^{k+1}}-\sum_{i=1}^{2 n}\left[\frac{B_{2 i}}{(2 i) !} \prod_{j=0}^{2 i-2}(-k-1-j)\right] \frac{1}{x^{2 i+k}},
$$

so that (7.2) and (7.5) imply

$$
\begin{aligned}
& \frac{1}{k !}(-1)^{k+1} F_{n}^{(k+1)}(x) \\
& \quad>\sum_{i=1}^{2 n}\left[\frac{(-1)^{k+1}}{k !} \frac{1}{2 i} \prod_{j=0}^{k-1}(-2 i-j)-\frac{1}{(2 i) !} \prod_{j=0}^{2 i-2}(-k-1-j)\right] \frac{B_{2 i}}{x^{2 i+k}}=0,
\end{aligned}
$$

since the term in square brackets is equal to 0 . Thus, $F_{n}$ is strictly completely monotonic on $(0, \infty)$.

Using the inequalities $(-1)^{k+1} F_{n}^{(k+1)}(x)>0$ and $(-1)^{k+1} G_{n}^{(k+1)}(x)>0$ for $k \geq 1$, we obtain the following rational bounds for $(-1)^{k+1} \psi^{(k)}(x)$.

Theorem 9. Let $k \geq 1$ and $n \geq 0$ be integers. Then we have for all real $x>0$ :

$$
S_{k}(2 n ; x)<(-1)^{k+1} \psi^{(k)}(x)<S_{k}(2 n+1 ; x),
$$

where

$$
S_{k}(p ; x)=\frac{(k-1) !}{x^{k}}+\frac{k !}{2 x^{k+1}}+\sum_{i=1}^{p}\left[B_{2 i} \prod_{j=1}^{k-1}(2 i+j)\right] \frac{1}{x^{2 i+k}} .
$$

Remark. Related inequalities for the special case $k=1$ are given in [22]. 
In 1986, J. Bustoz and M.E.H. Ismail [9] proved that the function

$$
p(x ; a, b)=\frac{\Gamma(x) \Gamma(x+a+b)}{\Gamma(x+a) \Gamma(x+b)} \quad(a, b>0)
$$

is completely monotonic on $(0, \infty)$. This generalizes a proposition of K. B. Stolarsky [46], who established that $p$ is decreasing in $x$. The next theorem provides an extension of these results.

Theorem 10. Let $a_{i}$ and $b_{i}(i=1, \ldots, n)$ be real numbers such that $0 \leq a_{1} \leq$ $\cdots \leq a_{n}, 0 \leq b_{1} \leq \cdots \leq b_{n}$, and $\sum_{i=1}^{k} a_{i} \leq \sum_{i=1}^{k} b_{i}$ for $k=1, \ldots, n$. Then,

$$
x \mapsto \prod_{i=1}^{n} \frac{\Gamma\left(x+a_{i}\right)}{\Gamma\left(x+b_{i}\right)}
$$

is completely monotonic on $(0, \infty)$.

In order to prove Theorem 10 we need the following two lemmas.

Lemma 1. If $h^{\prime}$ is completely monotonic on $(0, \infty)$, then $\exp (-h)$ is also completely monotonic on $(0, \infty)$.

An extension of Lemma 1 can be found in [5] and [15].

Lemma 2. Let $a_{i}$ and $b_{i}(i=1, \ldots, n)$ be real numbers such that $a_{1} \leq \cdots \leq a_{n}$, $b_{1} \leq \cdots \leq b_{n}$, and $\sum_{i=1}^{k} a_{i} \leq \sum_{i=1}^{k} b_{i}$ for $k=1, \ldots, n$. If the function $f$ is decreasing and convex on $\mathbb{R}$, then

$$
\sum_{i=1}^{n} f\left(b_{i}\right) \leq \sum_{i=1}^{n} f\left(a_{i}\right)
$$

A proof of Lemma 2 is given in [36, p. 10].

Proof of Theorem 10. Let

$$
h(x)=\sum_{i=1}^{n}\left(\log \Gamma\left(x+b_{i}\right)-\log \Gamma\left(x+a_{i}\right)\right) .
$$

Then we have for $k \geq 0$ :

$$
\left(h^{\prime}(x)\right)^{(k)}=\sum_{i=1}^{n}\left(\psi^{(k)}\left(x+b_{i}\right)-\psi^{(k)}\left(x+a_{i}\right)\right) .
$$

Using the integral representations

$$
\psi(z)=-\gamma+\int_{0}^{\infty} \frac{e^{-t}-e^{-t z}}{1-e^{-t}} d t \quad(z>0)
$$

and

$$
\psi^{(m)}(z)=(-1)^{m+1} \int_{0}^{\infty} \frac{e^{-t z} t^{m}}{1-e^{-t}} d t \quad(z>0 ; m=1,2, \ldots)
$$

(see [16, p. 802], [34, p. 16]), we obtain for $k \geq 0$ :

$$
(-1)^{k}\left(h^{\prime}(x)\right)^{(k)}=\int_{0}^{\infty} \frac{e^{-t x} t^{k}}{1-e^{-t}} \sum_{i=1}^{n}\left(e^{-t a_{i}}-e^{-t b_{i}}\right) d t .
$$


Since the function $z \mapsto e^{-t z}(t \geq 0)$ is decreasing and convex on $\mathbb{R}$, we conclude from Lemma 2 that $\sum_{i=1}^{n}\left(e^{-t a_{i}}-e^{-t b_{i}}\right) \geq 0$, so that (8.1) implies

$$
(-1)^{k}\left(h^{\prime}(x)\right)^{(k)} \geq 0 \text { for } x>0 \text { and } k \geq 0 .
$$

Hence, $h^{\prime}$ is completely monotonic on $(0, \infty)$. Applying Lemma 1 we obtain that

$$
\exp (-h(x))=\prod_{i=1}^{n} \frac{\Gamma\left(x+a_{i}\right)}{\Gamma\left(x+b_{i}\right)}
$$

is also completely monotonic on $(0, \infty)$.

Remark. Since

$$
\lim _{x \rightarrow \infty} \frac{\Gamma(x+a)}{\Gamma(x+b)} x^{b-a}=1,
$$

we conclude from Theorem 10 that the inequality

$$
\prod_{i=1}^{n} \frac{\Gamma\left(x+a_{i}\right)}{\Gamma\left(x+b_{i}\right)} \geq 1 \quad(x>0)
$$

holds for all real numbers $a_{i}$ and $b_{i}(i=1, \ldots, n)$ which satisfy $0 \leq a_{1} \leq \cdots \leq a_{n}$, $0 \leq b_{1} \leq \cdots \leq b_{n}, \sum_{i=1}^{k} a_{i} \leq \sum_{i=1}^{k} b_{i}$ for $k=1, \ldots, n-1$, and $\sum_{i=1}^{n} a_{i}=\sum_{i=1}^{n} b_{i}$. This generalizes an inequality given in [9].

In a recently published paper L. Maligranda et al. [35] established that the function

$$
x \mapsto \Gamma(x)^{n-1} \Gamma\left(x+\sum_{i=1}^{n} a_{i}\right) / \prod_{i=1}^{n} \Gamma\left(x+a_{i}\right)
$$

$\left(a_{i}>0 ; i=1, \ldots, n\right)$ is decreasing on $(0, \infty)$. From Theorem 10 we conclude that this function is not only decreasing, but even completely monotonic on $(0, \infty)$. The following theorem presents a slight extension of this result.

Theorem 11. Let $\alpha$ be a real number and let $a_{i}(i=1, \ldots, n ; n \geq 2)$ be positive real numbers. The function

$$
x \mapsto \Gamma(x)^{\alpha} \Gamma\left(x+\sum_{i=1}^{n} a_{i}\right) / \prod_{i=1}^{n} \Gamma\left(x+a_{i}\right)
$$

is strictly completely monotonic on $(0, \infty)$ if and only if $\alpha=n-1$.

Proof. Let

$$
p_{\alpha}(x)=\Gamma(x)^{\alpha} \Gamma(x+b) / \prod_{i=1}^{n} \Gamma\left(x+a_{i}\right)
$$

with $b=\sum_{i=1}^{n} a_{i}$. Slight modifications of the proof of Theorem 10 show that $p_{n-1}$ is strictly completely monotonic on $(0, \infty)$. We assume now that $p_{\alpha}$ is strictly completely monotonic on $(0, \infty)$. Then, $p_{\alpha}$ is decreasing, so that we obtain for $x>0$ :

$$
\frac{\partial}{\partial x} \log p_{\alpha}(x)=\alpha \psi(x)+\psi(x+b)-\sum_{i=1}^{n} \psi\left(x+a_{i}\right) \leq 0 .
$$


This implies for all sufficiently large $x$ :

$$
\alpha \leq \sum_{i=1}^{n} \frac{\psi\left(x+a_{i}\right)}{\psi(x)}-\frac{\psi(x+b)}{\psi(x)} .
$$

Since $p_{\alpha}$ is completely monotonic on $(0, \infty)$, we obtain

$$
\begin{aligned}
0 & \leq\left(p_{\alpha}(x)\right)^{-2}\left[p_{\alpha}(x) \frac{\partial^{2} p_{\alpha}(x)}{\partial x^{2}}-\left(\frac{\partial p_{\alpha}(x)}{\partial x}\right)^{2}\right] \\
& =\alpha \psi^{\prime}(x)+\psi^{\prime}(x+b)-\sum_{i=1}^{n} \psi^{\prime}\left(x+a_{i}\right) ;
\end{aligned}
$$

see [17]. Hence, we have for $x>0$ :

$$
\sum_{i=1}^{n} \frac{\psi^{\prime}\left(x+a_{i}\right)}{\psi^{\prime}(x)}-\frac{\psi^{\prime}(x+b)}{\psi^{\prime}(x)} \leq \alpha .
$$

Since

$$
\lim _{x \rightarrow \infty} \psi(x+A) / \psi(x)=\lim _{x \rightarrow \infty} \psi^{\prime}(x+A) / \psi^{\prime}(x)=1 \quad(A>0),
$$

we conclude from (8.2) and (8.3) that $\alpha=n-1$.

We conclude with an application to probability theory. A probability measure $d \mu$ is infinitely divisible if for every natural number $n$ there exists a probability measure $d \mu_{n}$ such that

$$
d \mu=d \mu_{n} * d \mu_{n} * \cdots * d \mu_{n} \quad(n \text { times }),
$$

where $*$ denotes convolution.

A proof for the following proposition, which provides a connection between infinitely divisible probability measures and completely monotonic functions, can found in $[15$, p. 450].

Proposition. A probability measure $d \mu$ supported on a subset of $[0, \infty)$ is infinitely divisible if and only if

$$
\int_{0}^{\infty} e^{-x t} d \mu(t)=\exp (-h(x)) \quad(x>0),
$$

where $h$ has a completely monotonic derivative on $(0, \infty)$ and $h(0)=0$.

Using the Proposition and the results of this section, we obtain

Theorem 12. Let $\varepsilon>0$ be a real number, and let $a_{i}$ and $b_{i}(i=1, \ldots, n)$ be real numbers such that $0 \leq a_{1} \leq \cdots \leq a_{n}, 0 \leq b_{1} \leq \cdots \leq b_{n}$, and $\sum_{i=1}^{k} a_{i} \leq \sum_{i=1}^{k} b_{i}$ for $k=1, \ldots, n$. The function

$$
x \mapsto \prod_{i=1}^{n} \frac{\Gamma\left(x+\varepsilon+a_{i}\right) \Gamma\left(\varepsilon+b_{i}\right)}{\Gamma\left(x+\varepsilon+b_{i}\right) \Gamma\left(\varepsilon+a_{i}\right)}
$$

is Laplace transform of an infinitely divisible probability measure.

Related results are given in [2], [9], [24].

\section{ACKNOWLEDGEMENT}

I thank the referee for bringing reference [22] to my attention. 


\section{REFERENCES}

1. M. Abramowitz and I. A. Stegun, eds., Handbook of mathematical functions with formulas, graphs and mathematical tables, Dover, New York, 1965. MR 31:1400

2. H. Alzer, Some gamma function inequalities, Math. Comp. 60 (1993), 337-346. MR 93f:33001

3. G. D. Anderson, R. W. Barnard, K. C. Richards, M. K. Vamanamurthy, and M. Vuorinen, Inequalities for zero-balanced hypergeometric functions, Trans. Amer. Math. Soc. 347 (1995), 1713-1723. MR 95m:33002

4. T. M. Apostol, Introduction to analytic number theory, Springer, New York, 1976. MR 55:7892

5. S. Bochner, Harmonic analysis and the theory of probability, Univ. of California Press, Berkeley/Los Angeles, 1955. MR 17:273d

6. A. V. Boyd, Gurland's inequality for the gamma function, Skand. Aktuarietidskr. 1960 (1961), 134-135. MR 24:A2058

7. __ Note on a paper by Uppuluri, Pacific J. Math. 22 (1967), 9-10. MR 35:6872

8. A. M. Bruckner and E. Ostrow, Some function classes related to the class of convex functions, Pacific J. Math. 12 (1962), 1203-1215. MR 26:6326

9. J. Bustoz and M. E. H. Ismail, On gamma function inequalities, Math. Comp. 47 (1986), 659-667. MR 87m:33002

10. P. J. Davis, Leonhard Euler's integral: A historical profile of the gamma function, Amer. Math. Monthly 66 (1959), 849-869. MR 21:5540

11. W. A. Day, On monotonicity of the relaxation functions of viscoelastic materials, Proc. Cambridge Philos. Soc. 67 (1970), 503-508. MR 40:3779

12. C. J. Eliezer and D. E. Daykin, Generalizations and applications of Cauchy-Schwarz inequalities, Quart. J. Math. Oxford Ser. (2) 18 (1967), 357-360. MR 37:1541

13. T. Erber, The gamma function inequalities of Gurland and Gautschi, Skand. Aktuarietidskr. 1960 (1961), 27-28. MR 24:A2682

14. A. Erdélyi, ed., Higher transcendental functions, vol. 1, McGraw-Hill, New York, 1953. MR 15:419i

15. W. Feller, An introduction to probability theory and its applications, Vol. 2, Wiley, New York, 1966. MR 35:1048

16. G. M. Fichtenholz, Differential- und Integralrechnung II, Dt. Verlag Wiss., Berlin, 1978. MR 80f: 26001

17. A. M. Fink, Kolmogorov-Landau inequalities for monotone functions, J. Math. Anal. Appl. 90 (1982), 251-258. MR 84e:26017

18. W. Gautschi, Some elementary inequalities relating to the gamma and incomplete gamma function, J. Math. Phys. 38 (1959), 77-81. MR 21:2067

19. - A harmonic mean inequality for the gamma function, SIAM J. Math. Anal. 5 (1974), 278-281. MR 50:2570

20. 282-292. MR 50:2571

21. D. V. Gokhale, On an inequality for gamma functions, Skand. Aktuarietidskr. 1962 (1963), 213-215. MR 28:4151

22. L. Gordon, A stochastic approach to the gamma function, Amer. Math. Monthly 101 (1994), 858-865. MR 95k:33003

23. J. Gurland, An inequality satisfied by the gamma function, Skand. Aktuarietidskr. 39 (1956), 171-172. MR 20:1797

24. M. E. H. Ismail, L. Lorch, and M. E. Muldoon, Completely monotonic functions associated with the gamma function and its q-analogues, J. Math. Anal. Appl. 116 (1986), 1-9. MR 88b:33002

25. J. D. Kečkić and P. M. Vasić, Some inequalities for the gamma function, Publ. Inst. Math. (Beograd) (N.S.) 11 (1971), 107-114. MR 46:7560

26. D. Kershaw, Some extensions of W. Gautschi's inequalities for the gamma function, Math. Comp. 41 (1983), 607-611. MR 84m:33003

27. D. Kershaw and A. Laforgia, Monotonicity results for the gamma function, Atti Accad. Sci. Torino 119 (1985), 127-133. MR 87i:33006

28. C. H. Kimberling, A probabilistic interpretation of complete monotonicity, Aequationes Math. 10 (1974), 152-164. MR 50:5899 
29. A. Laforgia, Further inequalities for the gamma function, Math. Comp. 42 (1984), 597-600. MR 85i:33001

30. I. B. Lazarević and A. Lupas, Functional equations for Wallis and gamma functions, Univ. Beograd. Publ. Elektrotehn. Fak. Ser. Electr. Telec. Autom. 461-497 (1979), 245-251. MR 50:13631

31. L. Lorch, Inequalities for ultraspherical polynomials and the gamma function, J. Approx. Theory 40 (1984), 115-120. MR 85d:33024

32. L. G. Lucht, Mittelwertungleichungen für Lösungen gewisser Differenzengleichungen, Aequationes Math. 39 (1990), 204-209. MR 91h:39004

33. Y. L. Luke, Inequalities for the gamma function and its logarithmic derivative, Math. Balkanica 2 (1972), 118-123. MR 50:10338

34. W. Magnus, F. Oberhettinger, and R. P. Soni, Formulas and theorems for the special functions of mathematical physics, Springer, Berlin, 1966. MR 38:1291

35. L. Maligranda, J. E. Pečarić, and L. E. Persson, Stolarsky's inequality with general weights, Proc. Amer. Math. Soc. 123 (1995), 2113-2118. MR 95i:26026

36. A. W. Marshall and I. Olkin, Inequalities: Theory of majorization and its applications, Academic Press, New York, 1979. MR 81b:00002

37. H. Minc and L. Sathre, Some inequalities involving $(r !)^{1 / r}$, Edinburgh Math. Soc. 14 (1964/65), 41-46. MR 29:55

38. D. S. Mitrinović, Analytic inequalities, Springer, New York, 1970. MR 43:448

39. M. E. Muldoon, Some monotonicity properties and characterizations of the gamma function, Aequationes Math. 18 (1978), 54-63. MR 58:11536

40. I. Olkin, An inequality satisfied by the gamma function, Skand. Aktuarietidskr. 1958 (1959), 37-39. MR 21:4257

41. H. Ruben, Variance bounds and orthogonal expansions in Hilbert space with an application to inequalities for gamma functions and $\pi$, J. Reine Angew. Math. 225 (1967), 147-153. MR 35: 421

42. J. Sándor, Sur la fonction gamma, Publ. C.R.M.P. Neuchâtel, Sér. I, 21 (1989), 4-7.

43. E. Schmidt, Über die Ungleichung, welche die Integrale über eine Potenz einer Funktion und über eine andere Potenz ihrer Ableitung verbindet, Math. Ann. 117 (1940), 301-326. MR 2:218e

44. J. B. Selliah, An inequality satisfied by the gamma function, Canad. Math. Bull. 19 (1976), 85-87. MR 54:3050

45. D. V. Slavić, On inequalities for $\Gamma(x+1) / \Gamma(x+1 / 2)$, Univ. Beograd. Publ. Elektrotehn. Fak. Ser. Mat. Fiz. 498-541 (1975), 17-20. MR 52:6047

46. K. B. Stolarsky, From Wythoff's Nim to Chebyshev's inequality, Amer. Math. Monthly 98 (1991), 889-900. MR 93b:90132

47. S. Y. Trimble, J. Wells, and F. T. Wright, Superadditive functions and a statistical application, SIAM J. Math. Anal. 20 (1989), 1255-1259. MR 91a:26019

48. D. V. Widder, The Laplace transform, Princeton Univ. Press, Princeton, 1941. MR 3:232d

49. J. Wimp, Sequence transformations and their applications, Academic Press, New York, 1981. MR 84e: 65005

50. S. Zimering, On a Mercerian theorem and its application to the equiconvergence of Cesàro and Riesz transforms, Publ. Inst. Math. (Beograd) (N.S.) 1 (1962), 83-91. MR 31:543

Morsbacher Str. 10, 51545 Waldbröl, Germany 